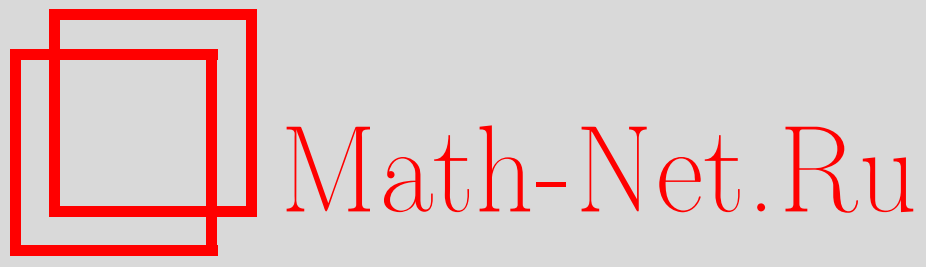

М. 3. Соломяк, О дискретном спектре семейства дифференциальных операторов, Функи. анализ и его прил., 2004, том 38, выпуск 3, 70-78

DOI: https://doi.org/10.4213/faa118

Использование Общероссийского математического портала MathNet.Ru подразумевает, что вы прочитали и согласны с пользовательским соглашением

http://www . mathnet.ru/rus/agreement

Параметры загрузки:

IP : 18.208 .226 .222

26 апреля 2023 г., 15:22:38

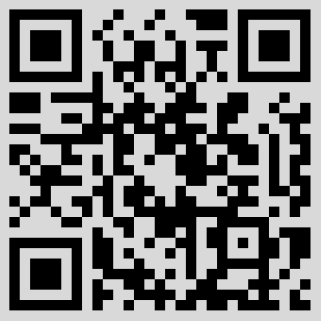


Функииональный анализ и его приложения

2004, т. 38, вып. 3, с. 70-78

УДК 517.97

\title{
О дискретном спектре семейства дифференциальных операторов
}

\author{
(c) 2004. М. 3. Соломяк
}

Посвящается Виктору Борисовичу Лидскому в связи с его восъмидесятилетием

\section{$\S 1$. Введение}

В настоящей работе мы изучаем дискретный спектр семейства $\mathbf{A}_{\alpha}$ дифференциальных операторов, определенных в пространстве $L^{2}\left(\mathbb{R}^{2}\right)$ дифференциальным выражением

$$
\mathscr{A} U=-U_{x x}^{\prime \prime}+\frac{1}{2}\left(-U_{y y}^{\prime \prime}+y^{2} U\right)
$$

и «условием сопряжения» на прямой $x=0$

$$
U_{x}^{\prime}(+0, y)-U_{x}^{\prime}(-0, y)=\alpha y U(0, y), \quad y \in \mathbb{R} .
$$

В формуле (1.2) через $\alpha$ обозначен вещественный параметр (константа связи). Таким образом, дифференциальное выражение, задающее действие оператора, не содержит параметра $\alpha$. Он появляется только в условии (1.2), которое задает область определения оператора $\mathbf{A}_{\alpha}$. Подстановка $\alpha \mapsto-\alpha$ соответствует замене переменных $y \mapsto-y$, которая не влияет на спектр. Поэтому в дальнейшем мы обсуждаем лишь случай $\alpha>0$.

Как мы увидим, спектр $\sigma\left(\mathbf{A}_{\alpha}\right)$ оператора $\mathbf{A}_{\alpha}$ имеет дискретную составляющую только при $\alpha<\sqrt{2}$, и мы изучим ее поведение при $\alpha \nearrow \sqrt{2}$.

Операторы, содержащие семейство $\mathbf{A}_{\alpha}$ в качестве частного случая, были введены Смилянским [8] как модель необратимой квантовой системы. Некоторые важные заключения о спектре семейства $\mathbf{A}_{\alpha}$ были сделаны в [8] «на физическом уровне строгости». Первые математические результаты на эту тему были получены в [9]. Они были существенно расширены в [7]. Работа [7] еще не опубликована, и ее результаты в данной статье не используются. Однако ниже мы упоминаем некоторые из этих результатов при обсуждении общих вопросов.

Для каждого $\alpha>0$ оператор $\mathbf{A}_{\alpha}$ допускает единственную естественную самосопряженную реализацию (см. [9] при $\alpha \neq \sqrt{2}$ и [7] при $\alpha=\sqrt{2}$ ). Выделенная роль значения $\alpha=\sqrt{2}$ будет объяснена ниже. Мы будем называть значения $\alpha<\sqrt{2}$ мальми, а значения $\alpha>\sqrt{2}$ большими. Спектральные свойства оператора $\mathbf{A}_{\alpha}$ для малых и больших значений параметра $\alpha$ значительно различаются между собой. 
Полезно рассмотреть квадратичную форму $\mathbf{a}_{\alpha}[U]$, которая формально соответствует оператору $\mathbf{A}_{\alpha}$. Она может быть записана в виде $\mathbf{a}_{\alpha}=\mathbf{a}_{0}+\alpha \mathbf{b}$, где

$$
\begin{gathered}
\mathbf{a}_{0}[U]=\int_{\mathbb{R}^{2}}\left(\left|U_{x}^{\prime}\right|^{2}+\frac{1}{2}\left(\left|U_{y}^{\prime}\right|^{2}+y^{2}|U|^{2}\right)\right) d x d y, \\
\mathbf{b}[U]=\int_{\mathbb{R}} y|U(0, y)|^{2} d y .
\end{gathered}
$$

Мы рассматриваем $\mathbf{a}_{0}[U]$ как невозмущенную квадратичную форму, а $\alpha \mathbf{b}[U]-$ как возмущение. Важная особенность рассматриваемой задачи проистекает из того факта, что форма $\mathbf{b}[U]$ только относительно ограничена, но не компактна относительно квадратичной формы $\mathbf{a}_{0}[U]$. По этой причине стандартные результаты теории возмущений неприменимы, что делает изучение операторов $\mathbf{A}_{\alpha}$ интересной и нетривиальной задачей.

Оказывается, что $\mathbf{a}_{0}$-грань квадратичной формы $\mathbf{b}[U]$ в точности равна $1 / \sqrt{2}$. Это объясняет роль пограничного значения $\alpha=\sqrt{2}$. Техника квадратичных форм неприменима к большим значениям параметра $\alpha$. В [7] (а отчасти уже и в [9]) доказано, что спектр оператора $\sigma\left(\mathbf{A}_{\alpha}\right)$ при $\alpha>\sqrt{2}$ является чисто непрерывным и совпадает со всей вещественной прямой. Спектр оператора $\mathbf{A}_{\sqrt{2}}$ также чисто непрерывен и совпадает с полупрямой $[0, \infty)$.

Оператор $\mathbf{A}_{0}$ легко исследовать с помощью разделения переменных. Он разлагается в ортогональную сумму операторов на пространстве $L^{2}(\mathbb{R})$, задаваемых формулой

$$
\mathbf{H}_{n}=-d^{2} / d x^{2}+(n+1 / 2), \quad n \in \mathbb{N}_{0}:=\{0,1, \ldots\} .
$$

Отсюда следует, что спектр $\sigma\left(\mathbf{A}_{0}\right)$ является абсолютно непрерывным и совпадает с полупрямой $[1 / 2, \infty)$. Его кратность равна $2 n$ на каждом интервале $(n-1 / 2, n+1 / 2), n \in \mathbb{N}$.

Следующее утверждение, описывающее спектральные свойства оператора $A_{\alpha}$ при малых $\alpha$, представляет собой частный случай теоремы 6.2 из [9].

ПреДЛОЖЕНИЕ 1.1. Пусть $\alpha<\sqrt{2}$. Тогда $\sigma_{\mathrm{ess}}\left(\mathbf{A}_{\alpha}\right)=\sigma\left(\mathbf{A}_{0}\right)=[1 / 2, \infty)$. Спектр оператора $\mathbf{A}_{\alpha}$ ниже порога $\lambda_{0}=1 / 2$ лежит в интервале $(0,1 / 2)$, всегда непуст и состоит из конечного числа собственных значений.

Такая структура нижнего спектра типична для относительно компактных возмущений, чего нет в нашем случае. Здесь работает и приводит к тому же результату (но только для малых значений параметра $\alpha$ ) другой механизм, который мы обсудим в заключительном $\S 5$.

Пусть задан самосопряженный оператор $\mathbf{T}$ в гильбертовом пространстве $\mathfrak{H}$ и вещественное число $s$. Введем обозначения

$$
N_{+}(s ; \mathbf{T})=\operatorname{dim} E^{\mathbf{T}}(s, \infty) \mathfrak{H}, \quad N_{-}(s ; \mathbf{T})=\operatorname{dim} E^{\mathbf{T}}(-\infty, s) \mathfrak{H},
$$

где $E^{\mathbf{T}}(\cdot)$ - спектральная мера оператора $\mathbf{T}$. Если, скажем, $N_{-}(s ; \mathbf{T})<\infty$, то спектр оператора $\mathbf{T}$ на интервале $(-\infty, s)$ сводится к конечному числу собственных значений (с учетом кратности) и $N_{-}(s ; \mathbf{T})$ равно числу этих значений.

Цель этой работы - изучить поведение функции $N_{-}\left(1 / 2 ; \mathbf{A}_{\alpha}\right)$ при $\alpha \nearrow \sqrt{2}$. Мы увидим, что $N_{-}\left(1 / 2 ; \mathbf{A}_{\alpha}\right) \rightarrow \infty$, и вычислим ее асимптотику. Этот результат дополняет предложение 1.1 количественной характеризацией дискретной части спектра $\sigma\left(\mathbf{A}_{\alpha}\right)$. Вероятно, центральным результатом статьи следует считать 
теорему 3.1. Она устанавливает равенство $N_{-}\left(1 / 2-\varepsilon ; \mathbf{A}_{\alpha}\right)=N_{+}(\sqrt{2} / \alpha ; \mathbf{J}(\varepsilon))$, где $\varepsilon \in(0,1 / 2)$ и $\mathbf{J}(\varepsilon)$ - некоторая матрица Якоби. Это аналог классического принципа Бирмана-Швингера для операторного семейства $\mathbf{A}_{\alpha}$.

\section{§2. Квадратичная форма $\mathbf{a}_{\alpha}$}

Квадратичная форма $\mathbf{a}_{0}$, задаваемая формулой (1.3), положительно определена и замкнута на своей естественной области определения

$$
D:=\operatorname{Dom} \mathbf{a}_{0}=\left\{U \in H^{1}\left(\mathbb{R}^{2}\right): \mathbf{a}_{0}[U]<\infty\right\},
$$

где, как обычно, $H^{1}$ - пространство Соболева. Самосопряженный оператор, порождаемый в $L^{2}\left(\mathbb{R}^{2}\right)$ квадратичной формой $\mathbf{a}_{0}[U]$, совпадает с $\mathbf{A}_{0}$, т. е. с оператором (1.1)-(1.2) при $\alpha=0$.

Удобно выразить обе квадратичные формы $\mathbf{a}_{0}$ и $\mathbf{b}$ в терминах разложения функции $U$ в ряд по (нормированным в $\left.L^{2}(\mathbb{R})\right)$ функциям Эрмита переменной $y$ :

$$
U(x, y)=\sum_{n \in \mathbb{N}_{0}} u_{n}(x) \chi_{n}(y) .
$$

Мы часто отождествляем функцию $U(x, y)$ с последовательностью $\left\{u_{n}(x)\right\}$ и пишем $U \sim\left\{u_{n}\right\}$. Это отождествление представляет собой унитарное отображение пространства $L^{2}\left(\mathbb{R}^{2}\right)$ на гильбертово пространство $\ell^{2}\left(\mathbb{N}_{0}, L^{2}(\mathbb{R})\right)$. Напомним рекуррентное соотношение для функций $\chi_{n}$ :

$$
\sqrt{n+1} \chi_{n+1}(y)-\sqrt{2} y \chi_{n}(y)+\sqrt{n} \chi_{n-1}(y)=0, \quad n \in \mathbb{N}_{0} .
$$

Подставляя в (1.3) представление (2.1) функции $U$, находим, что

$$
\mathbf{a}_{0}[U]=\sum_{n \in \mathbb{N}_{0}} \mathbf{h}_{n}\left[u_{n}\right]=: \sum_{n \in \mathbb{N}_{0}} \int_{\mathbb{R}}\left(\left|u_{n}^{\prime}\right|^{2}+\left(n+\frac{1}{2}\right)\left|u_{n}\right|^{2}\right) d x .
$$

Равенство (2.3) показывает, что разложение (2.1) диагонализует квадратичную форму $\mathbf{a}_{0}[U]$. Отсюда сразу вытекает разложение оператора $\mathbf{A}_{0}$ в ортогональную сумму операторов $\mathbf{H}_{n}$ (см. (1.5)), а значит, и описанная во введении структура спектра $\sigma\left(\mathbf{A}_{0}\right)$.

Таким же образом, принимая во внимание равенство (2.2), находим, что

$$
\mathbf{b}[U]=\sum_{n \in \mathbb{N}} \sqrt{2 n} \operatorname{Re}\left(u_{n}(0) \overline{u_{n-1}(0)}\right) .
$$

Лемма 2.1 (ср. $[9, \S 6])$. Квадратичная форма $\mathbf{b}[U]$ корректно определена на $D, u$

$$
\sqrt{2}|\mathbf{b}[U]| \leqslant \mathbf{a}_{0}[U] \quad \forall U \in D .
$$

ДокАЗАТЕЛЬСтво. Наши рассуждения основаны на неравенстве

$$
2 \gamma|u(0)|^{2} \leqslant \int_{\mathbb{R}}\left(\left|u^{\prime}\right|^{2}+\gamma^{2}|u|^{2}\right) d x \quad \forall u \in H^{1}(\mathbb{R}), \gamma>0,
$$

элементарное доказательство которого мы опустим. Легко также показать, что равенство в $(2.6)$ достигается на одномерном пространстве в $H^{1}(\mathbb{R})$, порожденном функцией

$$
\widetilde{u}_{\gamma}(x):=(2 \gamma)^{-1 / 2} e^{-\gamma|x|} .
$$


Здесь множитель $(2 \gamma)^{-1 / 2}$ подобран так, чтобы

$$
\int_{\mathbb{R}}\left(\left|\widetilde{u}_{\gamma}^{\prime}\right|^{2}+\gamma^{2}\left|\widetilde{u}_{\gamma}\right|^{2}\right) d x=1
$$

Из формулы (2.4) выводим, что

$$
\sqrt{2}|\mathbf{b}[U]| \leqslant \sum_{n \in \mathbb{N}} \sqrt{n}\left(\left|u_{n}(0)\right|^{2}+\left|u_{n-1}(0)\right|^{2}\right)=\sum_{n \in \mathbb{N}_{0}}(\sqrt{n}+\sqrt{n+1})\left|u_{n}(0)\right|^{2} .
$$

Поскольку $\sqrt{n}+\sqrt{n+1}<\sqrt{2(2 n+1)}$, мы заключаем из $(2.6)$, что

$$
\sqrt{2}|\mathbf{b}[U]| \leqslant \sum_{n \in \mathbb{N}_{0}} \mathbf{h}_{n}\left[u_{n}\right]=\mathbf{a}_{0}[U] \quad \forall U \sim\left\{u_{n}\right\} \in D,
$$

откуда следует (2.5).

Из леммы 2.1 вытекает, что при $0<\alpha<\sqrt{2}$ квадратичная форма $\mathbf{a}_{\alpha}[U]$ положительно определена:

$$
\mathbf{a}_{\alpha}[U] \geqslant\left(1-\frac{\alpha}{\sqrt{2}}\right) \mathbf{a}_{0}[U] \geqslant \frac{1}{2}\left(1-\frac{\alpha}{\sqrt{2}}\right)\|U\|^{2}, \quad U \in D
$$

(здесь и в дальнейшем $\left.\|U\|:=\|U\|_{L^{2}\left(\mathbb{R}^{2}\right)}\right)$. Кроме того, она замкнута (см., например, [2, Lemma 1.1]. Для таких $\alpha$ оператор $\mathbf{A}_{\alpha}$ может быть определен как самосопряженный оператор в $L^{2}\left(\mathbb{R}^{2}\right)$, ассоциированный с квадратичной формой $\mathbf{a}_{\alpha}[U]$.

\section{§3. Функция $N_{-}\left(1 / 2-\varepsilon ; A_{\alpha}\right)$}

Мы интересуемся нижним спектром оператора $\mathbf{A}_{\alpha}$, т. е. частью спектра, лежащей левее точки $1 / 2=\inf \sigma\left(\mathbf{A}_{0}\right)$. Согласно $(2.9)$, эта часть спектра $\sigma\left(\mathbf{A}_{\alpha}\right)$ содержится в интервале $1-\alpha / \sqrt{2} \leqslant 2 \lambda<1$. Общая теория возмущений не дает никакой дальнейшей информации, поскольку квадратичная форма b лишь относительно ограничена, но не компактна относительно формы $\mathbf{a}_{0}$. Однако мы сведем задачу к более простой для некоторого оператора Якоби в $\ell^{2}\left(\mathbb{N}_{0}\right)$. Эта редукция позволит нам решить исходную задачу.

Зафиксируем число $\varepsilon$ в интервале $0<\varepsilon<1 / 2$ и рассмотрим матрицу Якоби $\mathbf{J}(\varepsilon)$ с нулевой диагональю и с элементами

$$
j_{n, n-1}(\varepsilon)=j_{n-1, n}(\varepsilon)=\frac{n^{1 / 2}}{2(n+\varepsilon)^{1 / 4}(n-1+\varepsilon)^{1 / 4}}, \quad n \in \mathbb{N} .
$$

Все остальные элементы этой матрицы - нули. Тем же символом $\mathbf{J}(\varepsilon)$ обозначим и оператор в $\ell^{2}\left(\mathbb{N}_{0}\right)$, порожденный этой матрицей. Оператор $\mathbf{J}(\varepsilon)$ ограничен и самосопряжен, а его спектр инвариантен относительно отражения $\lambda \mapsto-\lambda$. Хорошо известно, что $\sigma_{\text {ac }}(\mathbf{J}(\varepsilon))=[-1,1]$. Кроме того, оператор может иметь (и действительно имеет) простые собственные значения $\pm \lambda_{n}, \lambda_{n}>1$, с единственно возможными точками накопления $\lambda= \pm 1$.

ТЕорема 3.1. Для каждого $\alpha \in(0, \sqrt{2})$ положим $s(\alpha)=\sqrt{2} / \alpha$. Тогда для любого $\varepsilon \in(0,1 / 2)$ справедливо равенство

$$
N_{-}\left(1 / 2-\varepsilon ; \mathbf{A}_{\alpha}\right)=N_{+}(s(\alpha), \mathbf{J}(\varepsilon))=N_{-}(-s(\alpha), \mathbf{J}(\varepsilon)) .
$$


Равенство (3.1) может рассматриваться как еще одно проявление общего принципа Бирмана-Швингера.

ДоКАЗАТЕЛЬСТВО. В соответствии с вариационным принципом имеем

$$
N_{-}\left(1 / 2-\varepsilon ; \mathbf{A}_{\alpha}\right)=\max _{\mathscr{F} \in \mathfrak{F}(\varepsilon)} \operatorname{dim} \mathscr{F},
$$

где $\mathfrak{F}(\varepsilon)-$ множество всех таких подпространств $\mathscr{F} \subset D$, что

$$
\mathbf{a}_{\alpha}[U]-(1 / 2-\varepsilon)\|U\|_{L^{2}\left(\mathbb{R}^{2}\right)}^{2}<0 \quad \forall U \in \mathscr{F}, U \neq 0 .
$$

Положим

$$
\|U\|_{\varepsilon}^{2}=\sum_{n \in \mathbb{N}_{0}} \int_{\mathbb{R}}\left(\left|u_{n}^{\prime}\right|^{2}+(n+\varepsilon)\left|u_{n}\right|^{2}\right) d x, \quad U \sim\left\{u_{n}\right\} .
$$

Для любых $\varepsilon>0$ и $U \in \mathfrak{H}$ квадратичная форма $\|U\|_{\varepsilon}^{2}$ оценивается через $\mathbf{a}_{0}[U]$ сверху и снизу и поэтому может быть взята в качестве формы, задающей метрику на $D$. Неравенство (3.3) можно переписать в виде

$$
\|U\|_{\varepsilon}^{2}+\alpha \sum_{n \in \mathbb{N}} \sqrt{2 n} \operatorname{Re}\left(u_{n}(0) \overline{u_{n-1}(0)}\right)<0 .
$$

Рассмотрим подпространство $\widetilde{D}(\varepsilon)$ в $D$, образованное элементами

$$
\widetilde{U} \sim\left\{C_{n} \widetilde{u}_{\sqrt{n+\varepsilon}}\right\}, \quad\left\{C_{n}\right\} \in \ell^{2}\left(\mathbb{N}_{0}\right),
$$

где элемент $\widetilde{u}_{\gamma}$ для любого $\gamma>0$ дается формулой (2.7). Заметим, что $\|\widetilde{U}\|_{\varepsilon}=$ $\left\|\left\{C_{n}\right\}\right\|_{\ell^{2}}$. Пусть $\Pi_{\varepsilon}-$ проектор в $D$ на $\widetilde{D}(\varepsilon)$, ортогональный относительно метрики $\|\cdot\|_{\varepsilon}$. Если $U \sim\left\{u_{n}\right\} \in D$, то

$$
\widetilde{U}_{\varepsilon}:=\Pi_{\varepsilon} U \sim\left\{C_{n} \widetilde{u}_{\sqrt{n+\varepsilon}}\right\},
$$

где

$$
C_{n}=\int_{\mathbb{R}}\left(u_{n}^{\prime} \widetilde{u}_{\sqrt{n+\varepsilon}}^{\prime}+(n+\varepsilon) u_{n} \widetilde{u}_{\sqrt{n+\varepsilon}}\right) d x=2^{1 / 2}(n+\varepsilon)^{1 / 4} u_{n}(0) .
$$

Мы видим, что

$$
C_{n} \widetilde{u}_{\sqrt{n+\varepsilon}}(0)=u_{n}(0) \quad \forall n \in \mathbb{N}_{0} .
$$

Если в неравенстве (3.4) заменить $U$ на $\widetilde{U}_{\varepsilon}$, то первый член в левой части не увеличится, а второй не изменится, так что неравенство останется верным. Другими словами, если подпространство $\mathscr{F} \subset D$ принадлежит классу $\mathfrak{F}(\varepsilon)$, то и $\Pi_{\varepsilon} \mathscr{F} \in \mathfrak{F}(\varepsilon)$.

С другой стороны, пусть $\mathscr{F}, \mathscr{F}^{\prime}-$ такие два подпространства класса $\mathfrak{F}(\varepsilon)$, что $\mathscr{F} \subset \mathscr{F}^{\prime}$ и $\mathscr{F} \subset \widetilde{D}(\varepsilon)$. Предположим, что существует элемент $U \sim\left\{u_{n}\right\} \in$ $\mathscr{F}^{\prime}$, ортогональный к $\mathscr{F}$ в $\varepsilon$-метрике. Тогда в силу (3.5) выполнено соотношение $u_{n}(0)=0$ для всех $n \in \mathbb{N}_{0}$. Отсюда следует, что $\mathbf{b}[U]=0$ в противоречии с (3.4). Таким образом, из нашего предположения вытекает, что $\mathscr{F}=\mathscr{F}^{\prime}$.

Из этих замечаний видно, что наряду с (3.2) имеет место равенство

$$
N_{-}\left(1 / 2-\varepsilon ; \mathbf{A}_{\alpha}\right)=\max _{\mathscr{F} \in \mathfrak{F}(\varepsilon), \mathscr{F} \subset \widetilde{D}(\varepsilon)} \operatorname{dim} \mathscr{F} .
$$


Для любого $\widetilde{U} \sim\left\{C_{n} \widetilde{u}_{\sqrt{n+\varepsilon}}\right\} \in \widetilde{D}(\varepsilon)$ имеем

$$
\begin{aligned}
\|\widetilde{U}\|_{\varepsilon}^{2}+\alpha \mathbf{b}[\widetilde{U}] & =\sum_{n \in \mathbb{N}_{0}}\left|C_{n}\right|^{2}+2 s^{-1} \sum_{n \in \mathbb{N}} j_{n, n-1}(\varepsilon) \operatorname{Re}\left(C_{n} \overline{C_{n-1}}\right) \\
& =\|g\|_{\ell^{2}}^{2}+s^{-1}(\mathbf{J}(\varepsilon) g, g)_{\ell^{2}}, \quad g=\left\{C_{n}\right\} \in \ell^{2} .
\end{aligned}
$$

Сумма в правой части есть не что иное, как квадратичная форма оператора $\mathbf{I}+s^{-1} \mathbf{J}(\varepsilon)$. Теперь равенство (3.1) следует из вариационного принципа и симметричности спектра $\sigma(\mathbf{J}(\varepsilon))$.

Теорема 3.1 неприменима к наиболее интересному случаю $\varepsilon=0$, поскольку $j_{1,0}(0)=\infty$. Однако мы можем ограничить квадратичную форму $(\mathbf{J}(\varepsilon) g, g)_{\ell^{2}}$ на подпространство $\left\{g=\left\{C_{n}\right\}: C_{0}=0\right\}$ коразмерности 1. При этом число собственных значений изменится, самое большее, на единицу. В полученной задаче переход к пределу при $\varepsilon \rightarrow 0$ уже возможен, и возникающая матрица Якоби с нулевой диагональю есть матрица $\mathbf{J}_{0}$ с внедиагональными элементами

$$
2 j_{n, n-1}=2 j_{n-1, n}=\left(1-n^{-1}\right)^{-1 / 4}, \quad n-1 \in \mathbb{N} .
$$

Итак, мы пришли к следующему результату.

Tеорема 3.2. Пусть $\alpha \in(0, \sqrt{2})$ and $s(\alpha)=\sqrt{2} / \alpha$. Тогда либо $N_{-}\left(1 / 2 ; \mathbf{A}_{\alpha}\right)$ $=N_{+}\left(s ; \mathbf{J}_{0}\right)$, либо $N_{-}\left(1 / 2 ; \mathbf{A}_{\alpha}\right)=N_{+}\left(s ; \mathbf{J}_{0}\right)+1$.

Благодаря теореме 3.2 задача об асимптотическом поведении функции $N_{-}\left(1 / 2 ; \mathbf{A}_{\alpha}\right)$ при $\alpha \nearrow \sqrt{2}$ сводится к вопросу об асимптотике собственных значений матрицы $\mathbf{J}_{0}$, лежащих правее точки $\lambda=1$. Мы не смогли найти соответствующий результат в литературе и потому выводим его в следующем параграфе. Вот его формулировка.

Теорема 3.3. Пусть J - матрица Якоби с нулевой диагональю и с внедиагональными элементами

$$
j_{n, n-1}=j_{n-1, n}=\frac{1}{2}+q n^{-1}(1+o(1)),
$$

где $q=$ const, $q>0$. Тогда оператор $\mathbf{J}$ имеет бесконечно много невырожденных собственных значений $\pm \lambda_{k}(\mathbf{J}), \operatorname{ma\kappa их,~что~}$

$$
\lambda_{k}(\mathbf{J})=1+\frac{2 q^{2}}{k^{2}}(1+o(1)), \quad k \rightarrow \infty .
$$

Эти собственные значения исчерпывают спектр оператора $\mathbf{J}$ вне отрезка $[-1,1]$. Равенство (3.8) можно эквивалентным образом записать в виде

$$
N_{+}(s ; \mathbf{J}) \sim \frac{q \sqrt{2}}{\sqrt{s-1}}, \quad s \searrow 1 .
$$

Из (3.6) следует, что элементы матрицы $\mathbf{J}_{0}$ удовлетворяют (3.7) при $q=1 / 8$. Поэтому из теорем 3.2 и 3.3 (равенство (3.9)) сразу же следует асимптотическая формула

$$
N_{-}\left(1 / 2 ; \mathbf{A}_{\alpha}\right) \sim \frac{1}{4 \sqrt{2(s(\alpha)-1)}}, \quad s(\alpha)=\frac{\sqrt{2}}{\alpha}, \alpha \searrow \sqrt{2}
$$




\section{§4. Доказательство теоремы 3.3}

Основным ингредиентом доказательства является результат ван Ассе [1] о классе ортогональных полиномов на вещественной оси, а именно, о так называемых полиномах Поллачека $P^{\lambda}(x ; a, b)$ (см., например, [3]). Они зависят от трех вещественных параметров $\lambda, a$ и $b$, но нам понадобится только их частный случай при $b=0, a=-r<0$ и $\lambda>r$. Нормируя полиномы $P^{\lambda}(x ;-r, 0)$ так, чтобы старший коэффициент стал равен единице, получаем новые полиномы, которые обозначим $Q^{\lambda}(x ; r)$. Они удовлетворяют рекуррентному соотношению

$$
\begin{gathered}
Q_{n+1}^{\lambda}(x ; r)=x Q_{n}^{\lambda}(x ; r)-p_{n}(\lambda, r) Q_{n-1}^{\lambda}(x ; r), \\
p_{n}(\lambda, r)=\frac{n(n+2 \lambda-1)}{4(n-r+\lambda-1)(n-r+\lambda)}, \quad n \in \mathbb{N} .
\end{gathered}
$$

Полиномы $Q_{n}^{\lambda}$ соответствуют матрице Якоби $\mathbf{J}(\lambda, r)$ с нулевой диагональю и внедиагональными элементами

$$
j_{n, n-1}=j_{n-1, n}=\sqrt{p_{n}(\lambda, r)} .
$$

$\mathrm{B}[1, \S \mathrm{III}]$ доказано, что спектр оператора $\mathbf{J}(\lambda, r)$ вне отрезка $[-1,1]$ состоит из невырожденных собственных значений $\pm \mu_{k}= \pm \mu_{k}(\lambda, r)$, где числа $\mu_{k}$ удовлетворяют уравнению

$$
\lambda-\frac{r \mu}{\sqrt{\mu^{2}-1}}=-k, \quad k \in \mathbb{N}_{0} .
$$

Отсюда получаем, что

$$
\mu_{k}=\left(1-\frac{r^{2}}{(k+\lambda)^{2}}\right)^{-1 / 2}=1+\frac{r^{2}}{2 k^{2}}+o\left(\frac{1}{k^{2}}\right), \quad k \rightarrow \infty .
$$

Другим ингредиентом является вариационный принцип для собственных значений матриц Якоби (см. [6, Lemma III.1]). Этот вариационный принцип - почти непосредственное следствие теоремы сравнения Штурма (см., например, [5, Theorem 1, p. 152]). Ниже мы даем формулировку для частного случая, который требуется в данной статье.

Лемма 4.1. Пусть $\mathbf{J}, \mathbf{J}^{\prime}$ - матрицы Якоби с нулевой диагональю и внедиагональными элементами $j_{n, n-1}=1 / 2+b_{n}, j_{n, n-1}^{\prime}=1 / 2+b_{n}^{\prime}$, такими, что $0 \leqslant b_{n} \leqslant b_{n}^{\prime}$ для всех $n$ u $b_{n}^{\prime} \rightarrow 0$. Тогда $\sigma_{\text {ess }}(\mathbf{J})=\sigma_{\text {ess }}\left(\mathbf{J}^{\prime}\right)=[-1,1] u$

$$
N^{+}(s ; \mathbf{J}) \leqslant N^{+}\left(s ; \mathbf{J}^{\prime}\right)
$$

для любого $s>1$.

Теперь мы можем доказать теорему 3.3. Из леммы 4.1 следует, что асимптотическое поведение собственных значений не зависит от слагаемого o(1) в (3.7). Элементы $j_{n, n-1}$ в (4.1) удовлетворяют соотношению $2 j_{n, n-1} \sim 1+r / n$. Очевидно, что равенство (3.8) является прямым следствием формулы (4.2).

\section{§5. Заключительные замечания}

5.1. Здесь мы объясним, почему при $\alpha<\sqrt{2}$ структура спектра $\sigma\left(\mathbf{A}_{\alpha}\right)$ оператора $\mathbf{A}_{\alpha}$ ниже порога $1 / 2$ такая же, как если бы возмущение было относительно компактным. Разумеется, одно из объяснений дается доказательством 
теоремы 3.1, но мы приведем еще одно рассуждение, в чем-то более эвристического характера. Строгая версия этого рассуждения использовалась в [9] при доказательстве теоремы 6.2.

Квадратичная форма $\mathbf{b}[U]$ (см. (1.4) и (2.4)) есть сумма членов

$$
\mathbf{b}_{n}[U]=\sqrt{2 n} \operatorname{Re}\left(u_{n}(0) \overline{u_{n-1}(0)}\right),
$$

каждый из которых имеет ранг два. Квадратичная форма $\mathbf{b}_{n}[U]$ взаимодействует только с членами $\mathbf{h}_{n-1}\left[u_{n-1}\right]$ и $\mathbf{h}_{n}\left[u_{n}\right]$ в представлении $(2.3)$ квадратичной формы $\mathbf{a}_{0}[U]$. Член $\mathbf{h}_{n}\left[u_{n}\right]$ соответствует оператору $\mathbf{H}_{n}$ (см. (1.5)), спектр которого есть $[n+1 / 2, \infty)$. Возмущение спектра, привнесенное членом $\alpha \mathbf{b}_{n}[U]$, не достигает точки $\lambda_{0}=1 / 2$ при условии, что $\alpha<\sqrt{2}$ и $n$ достаточно велико. Это означает, что, по существу, мы имеем дело с возмущением конечного ранга до тех пор, пока мы ограничиваемся малыми значениями константы связи и интересуемся только нижней частью спектра $\sigma\left(\mathbf{A}_{\alpha}\right)$.

5.2. В работе [9] операторное семейство $\mathbf{A}_{\alpha}$ было рассмотрено в более общей постановке. А именно, операторы действуют в пространстве $L^{2}(\Gamma \times \mathbb{R})$, где $\Gamma-$ метрический звездный граф, т. е. граф с $m$ ребрами $\mathscr{B}_{1}, \ldots, \mathscr{B}_{m}, 1 \leqslant m<\infty$, выходящими из общей вершины $о$. Напомним, что каждое ребро метрического графа рассматривается как отрезок прямой (конечный или бесконечный). Вещественная ось $\mathbb{R}$ может рассматриваться как звездный граф с двумя ребрами (так что $m=2$ ) бесконечной длины и вершиной $o=0$.

Отождествим каждое ребро $\mathscr{B}_{j}$ с отрезком $\left[0, B_{j}\right)$, где $B_{j} \leqslant \infty$ - длина ребра $\mathscr{B}_{j}$. Через $x$ обозначим координату вдоль каждого ребра (опуская индекс $j$ ); значение $x=0$ соответствует точке $o$.

Действие оператора $\mathbf{A}_{\alpha}$ в этом более общем случае определяется тем же равенством (1.1), в котором $y$ обозначает координату вдоль дополнительной прямой. Условие (1.2) заменяется условиями сопряжения

$$
U^{1}(0, y)=\cdots=U^{m}(0, y), \quad U_{x}^{1}(0, y)+\cdots+U_{x}^{m}(0, y)=\alpha y U(0, y),
$$

где $U^{j}$ обозначает ограничение функции $U$ на ребро $\mathscr{B}_{j}$. Кроме того, налагается условие Дирихле $U^{j}\left(B_{j}, y\right)=0$ на каждом ребре конечной длины.

Теорема 6.2 в [9] (ср. предложение 1.1 в данной статье) доказана для этой общей версии оператора $\mathbf{A}_{\alpha}$. Единственное отличие от частного случая $\Gamma=\mathbb{R}$ состоит в том, что а $\mathbf{a}_{0}$-грань квадратичной формы b равна $\sqrt{2} / m$ (cp. (2.5)). Соответственно техника, разработанная в данной статье, позволяет доказать аналог асимптотической формулы (3.10). Единственное отличие заключается в том, что для любого звездного графа с $m$ ребрами нужно взять $\alpha \nearrow m / \sqrt{2}$ и $s(\alpha)=m /(\alpha \sqrt{2})$.

В заключение я хотел бы выразить глубокую благодарность С. Н. Набоко за очень полезные обсуждения и рецензенту, указавшему на описку в формуле (3.8) в первоначальной версии статьи.

\section{ЛитерАТУРА}

1. Van Assche W. Pollaczek polynomials and summability methods. J. Math. Anal. Appl., 147, 498-505 (1990).

2. Birman M. Sh., Solomyak M. Z. Schrödinger operator. Estimates for number of bound states as function-theoretical problem. Amer. Math. Soc. Transl. Ser. 2, Vol. 150, Amer. Math. Soc., Providence, R.I., 1992, pp. 1-54. 
3. Chihara T. S. An introduction to orthogonal polynomials. Gordon and Breach, 1978.

4. Elaydi S. N. An introduction to difference equations. Springer-Verlag, New York, 1999.

5. Fort T. Finite difference equations in the real domain. Oxford University Press, 1948.

6. Geronimo J. S. On the spectra of infinite-dimensional Jacobi matrices. J. Approx. Theory, 53, 251-256 (1988).

7. Naboko S., Solomyak M. On the absolutely continuous spectrum of a family of operators appearing in the theory of irreversible quantum systems. Submitted to Proc. London Math. Soc.

8. Smilansky U. Irreversible quantum graphs. Waves Random Media, 14, 143-153 (2004).

9. Solomyak M. On a differential operator appearing in the theory of irreversible quantum graphs. Waves Random Media, 14, 173-185 (2004).

The Weizmann Institute of Science (Israel), Department of Mathematics

Поступило в редакцию email: michail.solomyak@weizmann.ac.il 30 января 2004 г. 